\title{
SHARP ESTIMATES FOR LEBESGUE CONSTANTS
}

\author{
MARCO CARENINI AND PAOLO M. SOARDI
}

\begin{abstract}
Suppose $C \subset R^{N}$ is a closed convex bounded body containing 0 in its interior. If $\partial C$ is sufficiently smooth with strictly positive Gauss curvature at each point, then, denoting by $L_{r, C}$ the Lebesgue constant relative to $C$, there exists a constant $A>0$ such that $L_{r, C} \geqslant A r^{(N-1) / 2}$ for $r$ sufficiently large. This complements the known result that there exists a constant $B$ such that $L_{r . C} \leqslant B r^{(N-1) / 2}$ for $r$ sufficiently large.
\end{abstract}

1. Let $R^{N}$ denote the $N$-dimensional euclidean space, $T^{N}$ the $N$-dimensional torus (identified with the cube $Q_{N}=\left\{t \in R^{N}:-\frac{1}{2} \leqslant t_{j}<\frac{1}{2}, j=1, \ldots, N\right\}$ ), and $Z^{N}$ the integer lattice of $R^{N}$. Throughout this paper we will assume $N \geqslant 2$. Suppose $C \subset R^{N}$ is a bounded closed convex body containing the origin in its interior and set $r C=\left\{x \in R^{N}: r^{-1} x \in C\right\}$ for every $r>0$. To any such $C$ we associate the Dirichlet kernel

$$
D_{r, C}(t)=\sum \exp (2 \pi i m t), \quad t \in T^{N},
$$

where the summation in (1) is extended to all $m \in r C \cap Z^{N}$. Sharp estimates for the Lebesgue constants

$$
L_{r, C}=\int_{T^{N}}\left|D_{r, C}(t)\right| d t
$$

are of interest in the study of norm convergence of multiple Fourier series. The aim of this paper is to prove there exists a positive constant $A$ such that $L_{r, C} \geqslant A r^{(N-1) / 2}$ for $r$ sufficiently large, whenever $C$ satisfies the regularity conditions of C. Herz [4] (this complements the known result that there exists $B>0$ such that $L_{r, C} \leqslant B r^{(N-1) / 2}$ for $r$ sufficiently large [5]). In fact our result is based on the asymptotic estimates of Herz for the Fourier transform of the characteristic function of $C$.

In the case where $C$ is a ball, the above result was proved earlier by $\mathrm{K}$. I. Babenko [2]. As the original paper is not readily accessible, and the proof given in [1] is complicated (as it deals with general eigenfunctions of the Laplacian), our theorem provides also a simple proof of the behaviour of the spherical Lebesgue constants.

As an application of our main result we extend to summations over $C$ the result of [3] on the best conditions for norm convergence of multiple Fourier series.

2. In the following we will always suppose $\partial C$ is of class $C^{k}$ with $k=[(N-1) / 2$ $+4]$, and the Gauss curvature of $\partial C$ is strictly positive at every point. We denote by

Received by the editors October 5. 1982.

1980 Mathematics Subject Classification. Primary 42B05, 42C99.

(C)1983 American Mathematical Society $0002-9939 / 83 \$ 1.00+\$ .25$ per page 
$H$ the supporting function of $C$ and set for all $t \in R^{N}$ :

$$
g(t)=\rho^{-(N-1) / 2} K(\theta)^{-1 / 2}\{\exp (2 \pi i(H(t)-(N-1) / 8))\} .
$$

Here we made $t=\rho \cdot \theta$, where $\rho \geqslant 0$ and $\theta$ is a norm 1 vector. $K(\theta)$ is the Gauss curvature at the point $x \in \partial C$ corresponding to $\theta$ under the inverse of the normal mapping.

THEOREM 1. If $C$ is as above and $L_{r, C}$ are defined by (2), then there are positive constants $A$ and $B$, depending only on $C$, such that as $r \rightarrow+\infty$,

$$
A r^{(N-1) / 2} \leqslant L_{r, C} \leqslant B r^{(N-1) / 2} \text {. }
$$

Proof. The right-hand inequality in (3) is a particular case of a theorem of Yudin [5]. Hence we have to prove only the left-hand inequality. Arguing as in [5 and 6],

$$
\int_{B_{r}} e^{2 \pi i t s} d s=\left(\prod_{j=1}^{N}\left(\pi t_{j}\right)^{-1} \sin \pi t_{j}\right) D_{r, C}(t),
$$

where $B_{r}$ denotes the union of all cubes of edge 1 centered at the points $m \in r C \cap Z^{N}$. Hence

$$
L_{r, C} \geqslant \int_{T^{N}}\left|\int_{B_{r}} e^{2 \pi i t s} d s\right| d t .
$$

Let $c_{r}$ denote the characteristic function of $r C$, and $b_{r}$ the characteristic function of $B_{r}$. Then $\left|b_{r}-c_{r}\right|$ is the characteristic function of the symmetric difference of $B_{r}$ and $r C$. For a small positive $\varepsilon$ (which will be chosen later) denote by $F_{\varepsilon}$ the annulus $F_{\varepsilon}=\left\{x \in R^{N}: \varepsilon \leqslant|x| \leqslant 2 \varepsilon\right\}$. Then, by (4),

$$
L_{r, C} \geqslant \int_{F_{r}}\left|\hat{c}_{r}(t)\right| d t-\int_{F_{t}}\left|\hat{b}_{r}(t)-\hat{c}_{r}(t)\right| d t=I_{1}-I_{2}
$$

The symmetric difference of $B_{r}$ and $r C$ is contained in the set of all points whose distance from $\partial r C$ is smaller than $2 N^{1 / 2}$. Hence, for big values of $r$ (depending only on $C$ ) we have $\left\|b_{r}-c_{r}\right\|_{2} \leqslant$ const $r^{(N-1) / 2}$. Therefore by the Schwarz inequality and the Plancherel theorem,

$$
I_{2} \leqslant M_{1} \varepsilon^{N / 2}\left\|b_{r}-c_{r}\right\|_{2} \leqslant M_{2} \varepsilon^{N / 2} r^{(N-1) / 2},
$$

where the constants $M_{1}$ and $M_{2}$ depend only on $C$.

On the other hand, Herz' asymptotic formula for $\hat{c}_{r}[4$, Theorem 3] reads

$$
2 \pi i \hat{c}_{r}(t)=r^{(N-1) / 2}\left\{(g(r t)-\overline{g(-r t)})|t|^{-1}\right\}+r^{N} O\left((r|t|)^{-(N+3) / 2}\right)
$$

where the error term depends only on $C$. Hence we have for, say, $r^{-1} \leqslant \varepsilon^{2}$,

$$
\begin{aligned}
r^{N} \int_{F_{\varepsilon}} O\left((r|t|)^{-(N+3) / 2}\right) d t & \leqslant M_{3} \varepsilon^{(N-3) / 2} r^{(N-3) / 2} \\
& \leqslant M_{3} \varepsilon^{(N+1) / 2} r^{(N-1) / 2}
\end{aligned}
$$


where the constant $M_{3}$ depends only on $C$. We set $f(\theta)=H(\theta)+H(-\theta)$. Then we get, by (7) and (8),

$$
\begin{aligned}
2 \pi I_{1} \geqslant & r^{(N-1) / 2} \int_{|\theta|=1} K(\theta)^{-1 / 2} d \theta \\
& \cdot \int_{\varepsilon}^{2 \varepsilon} \rho^{(N-3) / 2}|1-\exp -2 \pi i(r \rho f(\theta)-(N-1) / 4)| d \rho \\
& -M_{3} \varepsilon^{(N+1) / 2} r^{(N-1) / 2} \\
= & \int_{|\theta|=1} K(\theta)^{-1 / 2} f(\theta)^{-1} d \theta \\
& \cdot \int_{\varepsilon r f(\theta)}^{2 \varepsilon r f(\theta)} x^{(N-3) / 2}|1-\exp -2 \pi i(x-(N+1) / 4)| d x \\
& -M_{3} \varepsilon^{(N+1) / 2} r^{(N-1) / 2} .
\end{aligned}
$$

(We should remark that $f(\theta)>0$ since 0 belongs to the interior of $C$.)

Since $|1-\exp -2 \pi i(x-(N-1) / 4)|$ is periodic, for large values of $r(r>$ $10 \varepsilon^{-1} \max f(\theta)^{-1}$ will do) the inner integral is larger than

$$
M_{4} \sum_{j=h}^{k} j^{(N-3) / 2} \geqslant M_{5} \varepsilon^{(N-1) / 2} f(\theta)^{(N-1) / 2} r^{(N-1) / 2},
$$

where $h$ and $k$ are the integral parts of $r \varepsilon f(\theta)+1$ and $2 r \varepsilon f(\theta)$, respectively, and $M_{4}, M_{5}$ are constants independent of $r$ and $\varepsilon$. It follows, for large values of $r$ (depending only on $C$ and $\varepsilon$ ), that

$$
I_{1} \geqslant M_{6} \varepsilon^{(N-1) / 2} r^{(N-1) / 2}-M_{3} \varepsilon^{(N+1) / 2} r^{(N-1) / 2},
$$

where $M_{6}$ is a constant depending only on $C$. Now, choose $\varepsilon$ in such a way that

$$
M_{6} \varepsilon^{(N-1) / 2}-M_{3} \varepsilon^{(N+1) / 2}-M_{2} \varepsilon^{N / 2}>0 .
$$

Then the left-hand inequality in (3) follows from (6), (9) and (10).

The exact estimates of Theorem 1 allow an extension of Theorem A of [3]. Suppose $B$ is either the space $C\left(T^{N}\right)$ or the space $L^{1}\left(T^{N}\right)$. Denote by $\omega_{n}(\delta, f)$ the $n$th modulus of smoothness of a function $f$ in $B$. Then we have

THEOREM 2. Suppose $C$ is a closed, convex, bounded body satisfying the same assumptions as in Theorem 1. Then

(a) if $f \in B$ and $\omega_{n}(\delta, f)=o\left(\delta^{(N-1) / 2}\right)$ as $\delta \rightarrow 0$, for some $n>(N-1) / 2$, then

$$
\left\|D_{r, C} * f-f\right\|_{B} \rightarrow 0 \text { as } r \rightarrow \infty \text {; }
$$

(b) there exists a function $F \in B$ such that (for all $n>(N-1) / 2) \omega_{n}(\delta, F)=$ $O\left(\delta^{(N-1) / 2}\right)$ as $\delta \rightarrow 0$, but $D_{r, C} * F$ does not converge in norm as $r \rightarrow \infty$.

In other words the condition stated in (a) is the best condition, in terms of moduli of smoothness, for the norm convergence over $C$ of Fourier series in $B$. The proof of this theorem can be obtained by the same arguments as in [3]. 


\section{REFERENCES}

1. S. A. Alimov and V. A. Il'in, Conditions for the convergence of spectral decompositions that correspond to self-adjoint extensions of elliptic operators. I, II, Differential Equations 7 (1971), 516-543; 651-667.

2. K. I. Babenko, On the mean convergence of multiple Fourier series and the asymptotic behaviour of the Dirichlet kernel of the spherical means, Preprint No. 52, Inst. Prikl. Mat. Akad. Nauk SSSR, Moscow, 1971.

3. D. I. Cartwright and P. M. Soardi, Best conditions for the norm convergence of Fourier series, J. Approx. Theory (to appear).

4. C. S. Herz, Fourier transforms related to convex sets, Ann. of Math. (2) 75 (1962), 81-92.

5. V. A. Yudin, Behaviour of Lebesgue constants, Mat. Zametki 17 (1975), 401-405.

6. A. A. Yudin and V. A. Yudin, Discrete embedding theorems and Lebesgue constants, Mat. Zametki 22 (1977), 381-394.

Istituto Matematico dell'Universita, via C. Saldini 50, 20133 Milano, Italy 